\title{
Candidate Brown-dwarf Microlensing Events with Very Short Timescales and Small Angular Einstein Radii
}

\author{
Cheongho Han ${ }^{1}$ (1), Chung-Uk Lee ${ }^{2,32}$, Andrzej Udalski ${ }^{3,33}$, Andrew Gould ${ }^{4,5}$, Ian A. Bond ${ }^{6,34}$, Valerio Bozza ${ }^{7,8}$ (1) \\ (LEADING AUTHORS),
}

Michael D. Albrow ${ }^{9}$, Sun-Ju Chung ${ }^{2,10}$ (10, Kyu-Ha Hwang ${ }^{2}$ (1), Youn Kil Jung ${ }^{2}$, Yoon-Hyun Ryu ${ }^{2}$ (1) In-Gu Shin ${ }^{2}$ (1), Yossi Shvartzvald ${ }^{11}$ (1), Jennifer C. Yee ${ }^{12}$ (1), Weicheng Zang ${ }^{13}$ (1), Sang-Mok Cha ${ }^{2,14}$, Dong-Jin Kim ${ }^{2}$, Hyoun-Woo Kim ${ }^{2}$, Seung-Lee Kim ${ }^{2,10}$, Dong-Joo Lee ${ }^{2}$, Yongseok Lee ${ }^{2,14}$, Byeong-Gon Park ${ }^{2,10}$, Richard W. Pogge ${ }^{5}$ (1) M. James Jee ${ }^{15,16}$, Doeon $\mathrm{Kim}^{1}$

(The KMTNet Collaboration),

Przemek Mróz ${ }^{3,17}$, Michał K. Szymański ${ }^{3}$, Jan Skowron ${ }^{3}$ (10), Radek Poleski ${ }^{5}$, Igor Soszyński ${ }^{3}$, Paweł Pietrukowicz $^{3}$ (i), Szymon Kozłowski ${ }^{3}$, Krzysztof Ulaczyk ${ }^{18}$ (i) Krzysztof A. Rybicki $^{3}$, Patryk Iwanek ${ }^{3}$ (i), Marcin Wrona ${ }^{3}$

(The OGLE Collaboration),

and

Fumio Abe ${ }^{19}$, Richard Barry ${ }^{20}$, David P. Bennett ${ }^{20,21}$ (1) , Aparna Bhattacharya ${ }^{20,21}$, Martin Donachie ${ }^{22}$, Hirosane Fujii ${ }^{19}$, Akihiko Fukui $^{23,24}$ (1), Yoshitaka Itow ${ }^{19}$ (1), Yuki Hirao ${ }^{25}$, Yuhei Kamei ${ }^{19}$, Iona Kondo ${ }^{25}$ (1), Naoki Koshimoto ${ }^{26,27}$ (1), Man Cheung Alex Li ${ }^{22}$, Yutaka Matsubara ${ }^{19}$, Yasushi Muraki ${ }^{19}$, Shota Miyazaki ${ }^{25}$ (1), Masayuki Nagakane ${ }^{25}$, Clément Ranc ${ }^{20}$ (1), Nicholas J. Rattenbury ${ }^{22}$ (10), Yuki Satoh ${ }^{25}$, Hikaru Shoji ${ }^{25}$, Haruno Suematsu ${ }^{25}$, Denis J. Sullivan ${ }^{28}$, Takahiro Sumi ${ }^{25}$, Daisuke Suzuki $^{29}$ (1) , Paul J. Tristram ${ }^{30}$, Takeharu Yamakawa ${ }^{19}$, Tsubasa Yamawaki ${ }^{25}$, and Atsunori Yonehara ${ }^{31}$

(The MOA Collaboration)

${ }^{1}$ Department of Physics, Chungbuk National University, Cheongju 28644, Republic of Korea; cheongho@astroph.chungbuk.ac.kr

${ }^{2}$ Korea Astronomy and Space Science Institute, Daejon 34055, Republic of Korea

${ }^{3}$ Warsaw University Observatory, Al. Ujazdowskie 4, 00-478 Warszawa, Poland

${ }^{4}$ Max Planck Institute for Astronomy, Königstuhl 17, D-69117 Heidelberg, Germany

${ }^{5}$ Department of Astronomy, Ohio State University, 140 W. 18th Avenue, Columbus, OH 43210, USA

${ }^{6}$ Institute of Natural and Mathematical Sciences, Massey University, Auckland 0745, New Zealand

${ }^{7}$ Dipartimento di Fisica "E. R. Caianiello", Université di Salerno, Via Giovanni Paolo II, I-84084 Fisciano (SA), Italy

${ }^{9}$ University of Canterbury, Department of Physics and Astronomy, Private Bag 4800, Christchurch 8020, New Zealand

${ }^{10}$ Korea University of Science and Technology, 217 Gajeong-ro, Yuseong-gu, Daejeon, 34113, Republic of Korea

${ }^{11}$ Department of Particle Physics and Astrophysics, Weizmann Institute of Science, Rehovot 76100, Israel

${ }^{12}$ Center for Astrophysics|Harvard \& Smithsonian 60 Garden Street, Cambridge, MA 02138, USA

${ }^{13}$ Physics Department and Tsinghua Centre for Astrophysics, Tsinghua University, Beijing 100084, People's Republic of China

${ }^{14}$ School of Space Research, Kyung Hee University, Yongin, Kyeonggi 17104, Republic of Korea

${ }_{16}^{15}$ Yonsei University, Department of Astronomy, Seoul, Republic of Korea

${ }^{16}$ Department of Physics, University of California, Davis, California, USA

${ }^{17}$ Division of Physics, Mathematics, and Astronomy, California Institute of Technology, Pasadena, CA 91125, USA

${ }^{18}$ Department of Physics, University of Warwick, Gibbet Hill Road, Coventry, CV4 7AL, UK

${ }^{19}$ Institute for Space-Earth Environmental Research, Nagoya University, Nagoya 464-8601, Japan

${ }^{20}$ Code 667, NASA Goddard Space Flight Center, Greenbelt, MD 20771, USA

${ }^{21}$ Department of Astronomy, University of Maryland, College Park, MD 20742, USA

22 Department of Physics, University of Auckland, Private Bag 92019, Auckland, New Zealand

${ }^{23}$ Instituto de Astrofísica de Canarias, Vía Láctea s/n, E-38205 La Laguna, Tenerife, Spain

${ }^{24}$ Department of Earth and Planetary Science, Graduate School of Science, The University of Tokyo, 7-3-1 Hongo, Bunkyo-ku, Tokyo 113-0033, Japan

${ }^{25}$ Department of Earth and Space Science, Graduate School of Science, Osaka University, Toyonaka, Osaka 560-0043, Japan

${ }^{26}$ Department of Astronomy, Graduate School of Science, The University of Tokyo, 7-3-1 Hongo, Bunkyo-ku, Tokyo 113-0033, Japan

${ }^{27}$ National Astronomical Observatory of Japan, 2-21-1 Osawa, Mitaka, Tokyo 181-8588, Japan

${ }^{28}$ School of Chemical and Physical Sciences, Victoria University, Wellington, New Zealand

${ }^{29}$ Institute of Space and Astronautical Science, Japan Aerospace Exploration Agency, 3-1-1 Yoshinodai, Chuo, Sagamihara, Kanagawa, 252-5210, Japan

${ }^{30}$ University of Canterbury Mt. John Observatory, P.O. Box 56, Lake Tekapo 8770, New Zealand

${ }^{31}$ Department of Physics, Faculty of Science, Kyoto Sangyo University, 603-8555 Kyoto, Japan

Received 2019 October 24; revised 2020 January 22; accepted 2020 January 22; published 2020 February 28

\begin{abstract}
Short-timescale microlensing events are likely to be produced by substellar brown dwarfs (BDs), but it is difficult to securely identify BD lenses based on only event timescales $t_{\mathrm{E}}$ because short-timescale events can also be produced by stellar lenses with high relative lens-source proper motions. In this paper, we report three strong candidate BD-lens events found from the search for lensing events not only with short timescales $\left(t_{\mathrm{E}} \lesssim 6\right.$ days) but also with very small angular Einstein radii $\left(\theta_{\mathrm{E}} \lesssim 0.05\right.$ mas) among the events that have been found in the 2016-2019 observing seasons. These events include MOA-2017-BLG-147, MOA-2017-BLG-241, and MOA-
\end{abstract}

\footnotetext{
${ }^{32}$ KMTNet Collaboration.

33 OGLE Collaboration.

34 MOA Collaboration.
} 
2019-BLG-256, in which the first two events are produced by single lenses and the last event is produced by a binary lens. From the Monte Carlo simulations of Galactic events conducted with the combined $t_{\mathrm{E}}$ and $\theta_{\mathrm{E}}$ constraint, it is estimated that the lens masses of the individual events are $0.051_{-0.027}^{+0.100} M_{\odot}, 0.044_{-0.023}^{+0.090} M_{\odot}$, and $0.046_{-0.023}^{+0.067} M_{\odot} / 0.038_{-0.019}^{+0.056} M_{\odot}$ and the probability of the lens mass smaller than the lower limit of stars is $\sim 80 \%$ for all events. We point out that routine lens mass measurements of short-timescale lensing events require surveymode space-based observations.

Unified Astronomy Thesaurus concepts: Gravitational microlensing (672); Brown dwarfs (185)

\section{Introduction}

Considering that brown dwarfs (BDs) share a similar formation mechanism to that of their heavier-mass sibling stars (Whitworth et al. 2007) and the number of stars increases as their mass decreases (Chabrier et al. 2014), it may be that the Galaxy is teeming with BDs. Due to the intrinsic faintness, however, it is difficult to detect BDs from imaging or spectroscopic observations in optical wavelengths. Some BDs can be observed in infrared, e.g., McLean et al. (2003, 2007), but these observations are confined to nearby and relatively young and/or massive BDs. Microlensing provides an important method to detect BDs because the lensing phenomenon occurs by the gravity of lens objects regardless of their brightness.

In order to firmly identify $\mathrm{BD}$ lenses, it is required to determine lens masses. For general lensing events, the only observable related to the lens mass is the event timescale $t_{\mathrm{E}}$. The event timescale is related to the physical lens parameters by

$$
t_{\mathrm{E}}=\frac{\theta_{\mathrm{E}}}{\mu} ; \quad \theta_{\mathrm{E}}=\left(\kappa M \pi_{\mathrm{rel}}\right)^{1 / 2} ; \quad \pi_{\mathrm{rel}}=\mathrm{au}\left(\frac{1}{D_{\mathrm{L}}}-\frac{1}{D_{\mathrm{S}}}\right)
$$

where $\theta_{\mathrm{E}}$ is the angular Einstein radius, $\mu$ is the relative lenssource proper motion, $\kappa=4 G /\left(c^{2} \mathrm{au}\right), M$ is the lens mass, and $D_{\mathrm{L}}$ and $D_{\mathrm{S}}$ represent the distances to the lens and source, respectively. Because the timescale is proportional to the square root of the lens mass, i.e., $t_{\mathrm{E}} \propto \sqrt{M}$, a considerable fraction of events with very short timescales is likely to be produced by BDs. However, short-timescale events can also be produced by stellar lenses with high relative lens-source proper motions. Therefore, it is difficult to firmly identify BD lenses just based on the event timescale.

For a fraction of lensing events, it is possible to determine the angular Einstein radius, which is an additional observable related to the lens mass. The angular Einstein radius can be measured for events in which lensing lightcurves are affected by finite-source effects. For events with a single lens and a single source (1L1S events), these effects occur when the lens passes over the surface of a source star (Gould 1994a). See example events in Choi et al. (2012). For binary lens (2L1S) events, lensing lightcurves are affected by finite-source effects when the source passes over the caustic. Analysis of the lightcurve affected by finite-source effects yields the normalized angular source radius $\rho$, which is related to the angular Einstein radius and angular source radius $\theta_{*}$ by $\rho=\theta_{*} / \theta_{\mathrm{E}}$. Then, the angular Einstein radius is determined with the additional information of the angular source radius by $\theta_{\mathrm{E}}=\theta_{*} / \rho$. While the event timescale is related to the three parameters of $\mu, \pi_{\text {rel }}$, and $M$, the angular Einstein radius is related to only the two parameters of $\pi_{\text {rel }}$ and $M$. Therefore, the lens mass can be better constrained with the additionally measured value of $\theta_{\mathrm{E}}$.

With the increasing observational cadence of microlensing surveys, the number of events with additionally measured angular Einstein radii is rapidly increasing. The duration of finite-source effects is approximately

$$
\Delta t \sim \frac{2 \theta_{*}}{\mu} .
$$

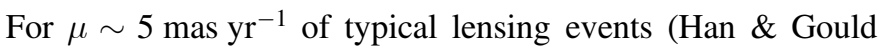
1995), the duration is on the order of hours for events associated with main-sequence source stars and $\sim 1$ day for events that occurred on giant source stars. With the observational cadence of $\sim 1$ day in the early stage of microlensing experiments, it was difficult to determine $\theta_{\mathrm{E}}$ by resolving the short-lasting parts of lensing lightcurves affected by finitesource effects. With the utilization of wide-field cameras together with the employment of globally distributed multiple telescopes, the observational cadence of lensing surveys has dramatically increased. This enables us to resolve finite-source lightcurves and determine angular Einstein radii for a greatly increased number of events.

In this paper, we present the analyses of three microlensing events that are very likely to be produced by BD lenses. For these events, the high probability of the BD-lens nature is identified not only by the short timescales but also by the very small angular Einstein radii.

The paper is organized as follows. In Section 2, we outline the procedure of selecting events analyzed in this work. In Section 3, we describe the observations of the events and the data acquired from the observations. We describe modeling the lightcurves of the individual events in Section 4 and mention the procedure of measuring the angular Einstein radii in Section 5. We estimate the masses and locations of the lenses in Section 6. In Section 7, we discuss the feasibility of measuring the microlens parallax for events similar to the analyzed events. We summarize the results and conclude in Section 8.

\section{Event Selection}

We search for candidate BD-lens events from the sample of lensing events that have been found in the 2016-2019 observing seasons. The 2016 season corresponds to the time of the full-scale operation of the current high-cadence lensing surveys: Optical Gravitational Lensing Experiment (OGLE: Udalski et al. 2015), Microlensing Observations in Astrophysics (MOA: Bond et al. 2001), and Korea Microlensing Telescope Net-work (KMTNet: Kim et al. 2016). During this period, more than 2000 events have been detected each year.

Selection of candidate BD-lens events are based on the combined information of the event timescale and the angular 
Einstein radius. For this, we first pick out short-timescale events, for which finite-source deviations in lensing lightcurves are detected. In the second step, we select events with very small angular Einstein radii. Rough estimation of $t_{\mathrm{E}}$ can be easily done from the durations of events. In contrast, estimating $\theta_{\mathrm{E}}$ requires extra information of the source color, from which the angular source radius $\theta_{*}$ is estimated, and thus it is difficult to inspect a large sample of finite-source events. For the efficient search for events with very small $\theta_{\mathrm{E}}$, we inspect events that are affected by severe finite-source effects with very large normalized source radius $\rho$. This criterion is applied because the angular Einstein radius is related to the normalized source radius by $\theta_{\mathrm{E}}=\theta_{*} / \rho$, and thus a large $\rho$ value suggests that $\theta_{\mathrm{E}}$ is likely to be small. We note that the shortcoming of this criterion is that it tends to restrict to source stars with large angular radii, i.e., giant stars, and thus limits the sample. For this reason, we note that there could be more events with small $\theta_{\mathrm{E}}$ from the events with lower-luminosity source stars.

In the selection of events, we impose requirements of $t_{\mathrm{E}} \lesssim 6$ days and $\rho \gtrsim \rho_{\text {th }} \equiv 0.1$. We note that the imposed threshold value $\rho_{\text {th }}=0.1$ is much greater than typical values of events associated with main-sequence stars, $\sim(O) 10^{-3}$, and giant stars, $\sim(O) 10^{-2}$. For events that meet these requirements, we then estimate the angular Einstein radii and apply another criterion of $\theta_{\mathrm{E}}<0.05$ mas. ${ }^{35}$ From this procedure, we find three candidate BD-lens events, including MOA-2017-BLG147, MOA-2017-BLG-241, and MOA-2019-BLG-256, analyzed in this work. We note that MOA-2017-BLG-147 and MOA-2017-BLG-241 are 1L1S events and MOA-2019-BLG256 is a $2 \mathrm{~L} 1 \mathrm{~S}$ event.

We note that there are three more events satisfying the imposed criteria besides the events analyzed in this work. These events are OGLE-2016-BLG-1227, OGLE-2016-BLG1540, and OGLE-2017-BLG-0560. The lightcurve of the event OGLE-2016-BLG-1227 appears to be a 1L1S event affected by severe finite-source effects and the preliminary $1 \mathrm{~L} 1 \mathrm{~S}$ modeling yields $t_{\mathrm{E}} \sim 3.5$ days and $\theta_{\mathrm{E}} \sim 0.009$ mas, making the lens a strong candidate of either a BD or a free-floating planet. From detailed investigation, it is found that the event is produced by a wide-separation planet and the analyses will be presented in a separate paper. The events OGLE-2016-BLG-1540 (with $t_{\mathrm{E}} \sim 0.32$ days and $\theta_{\mathrm{E}} \sim 0.009$ mas) and OGLE-2017-BLG0560 (with $t_{\mathrm{E}} \sim 0.91$ days and $\theta_{\mathrm{E}} \sim 0.038$ mas) were analyzed by Mróz et al. (2018, 2019), respectively. They pointed out that the lens of OGLE-2016-BLG-1540 was likely to be a Neptunemass free-floating planet in the Galactic disk and the lens of OGLE-2017-BLG-0560 is either a Jupiter-mass free-floating planet in the disk or a BD in the bulge.

\section{Observations and Data}

The analyzed lensing events share a common observational property that the lightcurves of the events are densely observed by the major lensing surveys despite of their short timescales. All of the events are detected toward the Galactic bulge field. In Table 1, we list the positions of the events in the equatorial coordinate system, (R.A., decl.) J2000. Also listed are the galactic coordinates $(l, b)$ to indicate the positions of the events with respect to the Galactic center and plane. The first

\footnotetext{
35 For comparison, we note that the angular Einstein radius of a lensing event produced by a low-mass star with $M \sim 0.3 M_{\odot}$ located halfway between a source in the bulge and the observer is about $\theta_{\mathrm{E}} \sim 0.5$ mas.
}

column lists the event names. For each event, different names are given by the individual surveys, and we list all the names according to the chronological order of the event discovery. Hereafter, we use the names given by the first discovery survey as the representative names of the events.

The survey observations were conducted using multiple telescopes that were equipped with wide-field cameras and globally distributed in the southern hemisphere. The telescope used for the OGLE survey is located at the Las Campanas Observatory in Chile. The telescope has a $1.3 \mathrm{~m}$ aperture, and it is equipped with a mosaic camera that consists of 32 chips with each chip composed of $2 \mathrm{k} \times 4 \mathrm{k}$ pixels. The camera covers a $1.4 \mathrm{deg}^{2}$ field of view with a single exposure. The MOA $1.8 \mathrm{~m}$ telescope, located at the Mt. John Observatory in New Zealand, is equipped with a camera that consists of $102 \mathrm{k} \times 2 \mathrm{k}$ chips with a total $2.2 \mathrm{deg}^{2}$ field of view. The KMTNet observations were carried out using three identical $1.6 \mathrm{~m}$ telescopes located at the Siding Spring Observatory in Australia (KMTA), Cerro Tololo Interamerican Observatory in Chile (KMTC), and the South African Astronomical Observatory in South Africa (KMTS). The camera mounted on each of the KMTNet telescopes consists of four $9 \mathrm{k} \times 9 \mathrm{k}$ chips with a total $4 \mathrm{deg}^{2}$ field of view. The wide field of view of the surveys using the globally distributed telescopes enable dense and continuous coverage of the events despite their short durations. Observations by the OGLE and KMTNet surveys were conducted mostly in $I$ band with occasional observations in $V$ band. MOA observations were carried out in a customized broad $R / I$ filter.

Reduction of the data sets is conducted using the photometry codes developed by the individual survey groups based on the difference imaging method (Alard \& Lupton 1998): Woźniak (2000; OGLE), Bond et al. (2001; MOA), and Albrow et al. (2009; KMTNet). For a subset of the KMTC data set, additional photometry is conducted using the pyDIA code (Albrow 2017) for the source color measurement. We readjust the error bars of the individual data sets following the method described in Yee et al. (2012).

In Figures 1-3, we present the lightcurves of the MOA2017-BLG-147, MOA-2017-BLG-241, and MOA-2019-BLG256 , respectively. We note that the data points are linearly aligned with the OGLE data. As mentioned, the lightcurves of all events are affected by severe finite-source effects, and the peak regions show strong deviations from the point-source lightcurves (dashed curves). To better show the lightcurve deviation affected by finite-source effects, we present the zoom of the peak region in the upper panel of each figure. At first glance, the lightcurve of MOA-2019-BLG-256 appears to be similar to those of the other events produced by finite-source $1 \mathrm{~L} 1 \mathrm{~S}$ events, but a close look shows asymmetry with respect to the peak. As we will show in the following section, the event is produced by a binary lens.

\section{Modeling Lightcurves}

The first step for the analyses of the events is conducting modeling on the observed lightcurves. Lightcurve modeling is carried out by searching for a set of the lensing parameters that best describes the observed lightcurves. For a 1L1S event with a point source, the lensing lightcurve is described by three parameters of $t_{0}, u_{0}$, and $t_{\mathrm{E}}$ (Paczyński 1986). The first two of these parameters represent the time of the closest lens-source approach and the lens-source separation (normalized to $\theta_{\mathrm{E}}$ ) at that time, i.e., impact parameter, respectively. For a $1 \mathrm{~L} 1 \mathrm{~S}$ event 
Table 1

Coordinates of Events

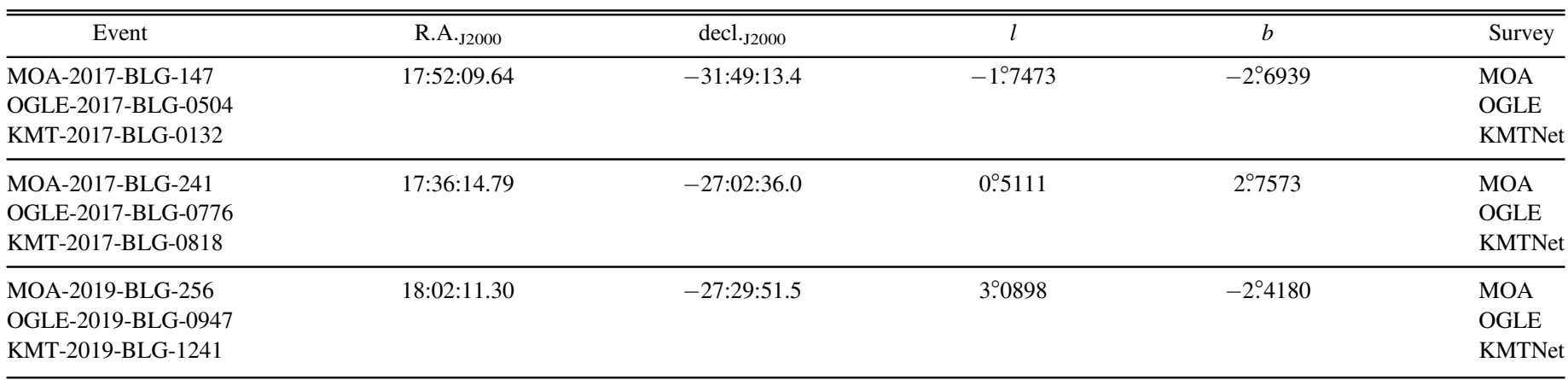

Note. For a single event, there are multiple names given by the individual surveys and the names are listed according to the chronological order of the event discovery. Hereafter we use the names given by the first discovery survey as the representative names of the events.
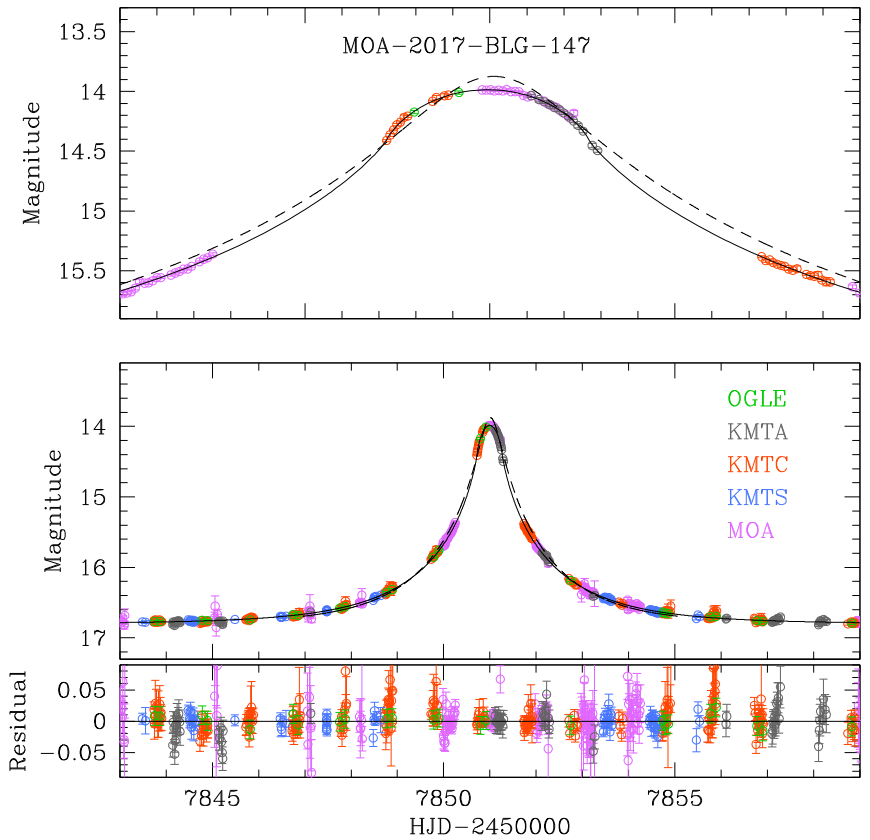

Figure 1. Lightcurve of MOA-2017-BLG-147. The middle panel shows the whole range of lensing magnification and the top panel shows the zoom of the peak region. The solid and dashed curves superposed on the data points represent the model curves obtained with and without considering finite-source effects, respectively. The colors of the data points are set to match those of the telescopes in the legend used for the data acquisition. The bottom panel shows the residual from the model considering finite-source effects.

in which the source radius is greater than the impact parameter, i.e., $\rho>u_{0}$, the lensing lightcurve is affected by finite-source effects. For the description of such events, one needs an additional lensing parameter of $\rho$. For 2L1S events, one needs additional parameters to describe the binary nature of the lens. These additional parameters include $s, q$, and $\alpha$. The parameter $s$ denotes the projected separation between the binary lens components, $M_{1}$ and $M_{2}<M_{1}$, and its length is scaled to the angular Einstein radius. The parameter $q$ represents the mass ratio between the binary lens components, i.e., $q=M_{2} / M_{1}$, and $\alpha$ represents the incidence angle of the source trajectory with respect to the $M_{1}-M_{2}$ axis.

Lensing magnifications affected by finite-source effects differ from those of a point source. For 1L1S events, we compute finite-source magnifications using the semianalytic expressions first derived by Gould (1994a) and Witt \&

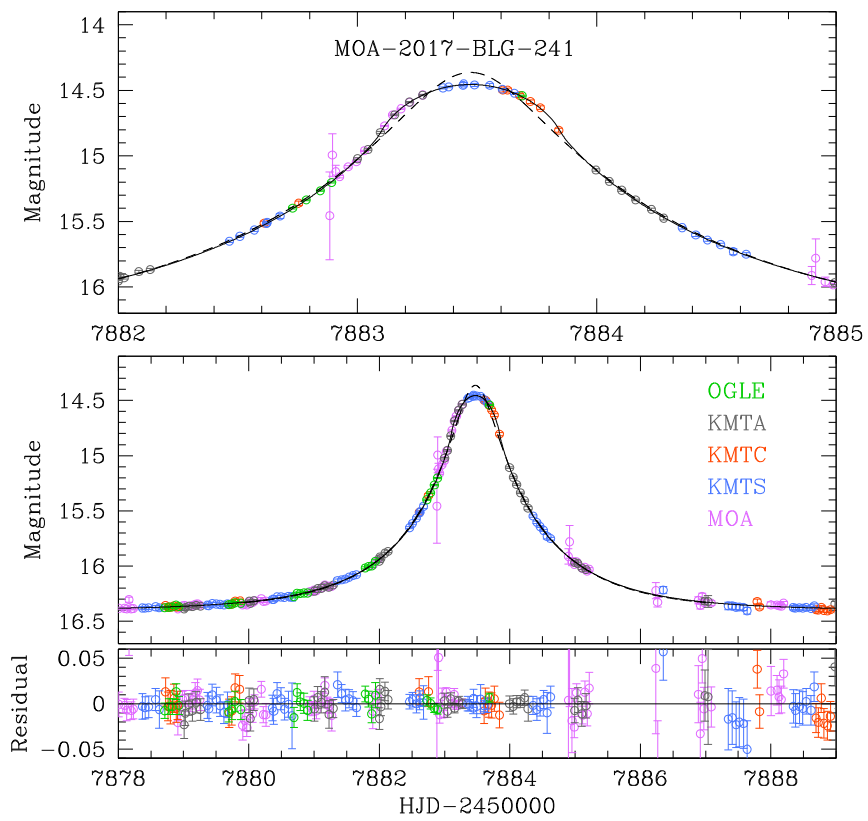

Figure 2. Lightcurve of MOA-2017-BLG-241. Notations are the same as those in Figure 1.

Mao (1994) and later refined by Yoo et al. (2004). These approximations may not be valid in the region of a very large $\rho$, and thus we check the validity of the expressions by comparing magnifications computed by using a contouring method. We find that the semianalytic expressions are valid in the cases of the analyzed events. For 2L1S events, we compute magnifications using the numerical ray-shooting method described in Dong et al. (2006). In computing finite-source magnifications, we consider the variation of the source surface brightness caused by limb darkening. To account for the limb-darkening variation, we model the surface brightness of the source star as

$$
S_{\lambda}=\bar{S}_{\lambda}\left[1-\Gamma_{\lambda}\left(1-\frac{3}{2} \cos \theta\right)\right] .
$$

Here $\bar{S}_{\lambda}$ denotes the mean surface brightness, $\Gamma_{\lambda}$ is the linear limb-darkening coefficient, and $\theta$ represents the angle between the line of sight toward the source center and the normal to the source surface. The limb-darkening coefficients are estimated based on the stellar types of the source stars. As we will show in the following section, the source stars of the analyzed events are 
Table 2

Best-fit Lensing Parameters

\begin{tabular}{lccc}
\hline \hline Parameter & MOA-2017-BLG-147 & MOA-2017-BLG-241 & MOA-2019-BLG-256 \\
\hline$t_{0}\left(\mathrm{HJD}^{\prime}\right)$ & $7850.994 \pm 0.001$ & $7883.473 \pm 0.001$ & $8662.089 \pm 0.001$ \\
$u_{0}$ & $0.092 \pm 0.001$ & $0.211 \pm 0.005$ & $0.076 \pm 0.001$ \\
$t_{\mathrm{E}}$ (days) & $2.679 \pm 0.023$ & $1.868 \pm 0.023$ & $8.723 \pm 0.008$ \\
$t_{\mathrm{E}, 1}$ (days) & $\ldots$ & $\ldots$ & $6.439 \pm 0.006$ \\
$t_{\mathrm{E}, 2}$ (days) & $\ldots$ & $\ldots$ & $5.884 \pm 0.005$ \\
$s$ & $\ldots$ & $\ldots$ & $1.968 \pm 0.002$ \\
$q$ & $\ldots$ & $\ldots$ & $0.835 \pm 0.003$ \\
$\alpha(\mathrm{rad})$ & $\ldots$ & $0.290 \pm 0.005$ & $2.313 \pm 0.001$ \\
$\rho$ & $0.137 \pm 0.001$ & 4.345 & $0.213 \pm 0.001$ \\
$f_{\mathrm{s}, \text { OGLE }}$ & 3.076 & 0.010 & 21.310 \\
$f_{\mathrm{b}, \text { OGLE }}$ & -0.062 & & -1.296 \\
\hline
\end{tabular}

Note. $\mathrm{HJD}^{\prime}=\mathrm{HJD}-2,450,000$. For the 2L1S event MOA-2019-BLG-256, $t_{\mathrm{E}}$ is the event timescale corresponding to the total mass of the binary lens, and $t_{\mathrm{E}, 1}$ and $t_{\mathrm{E}, 2}$ represent the timescales corresponding to the masses of individual lens components.

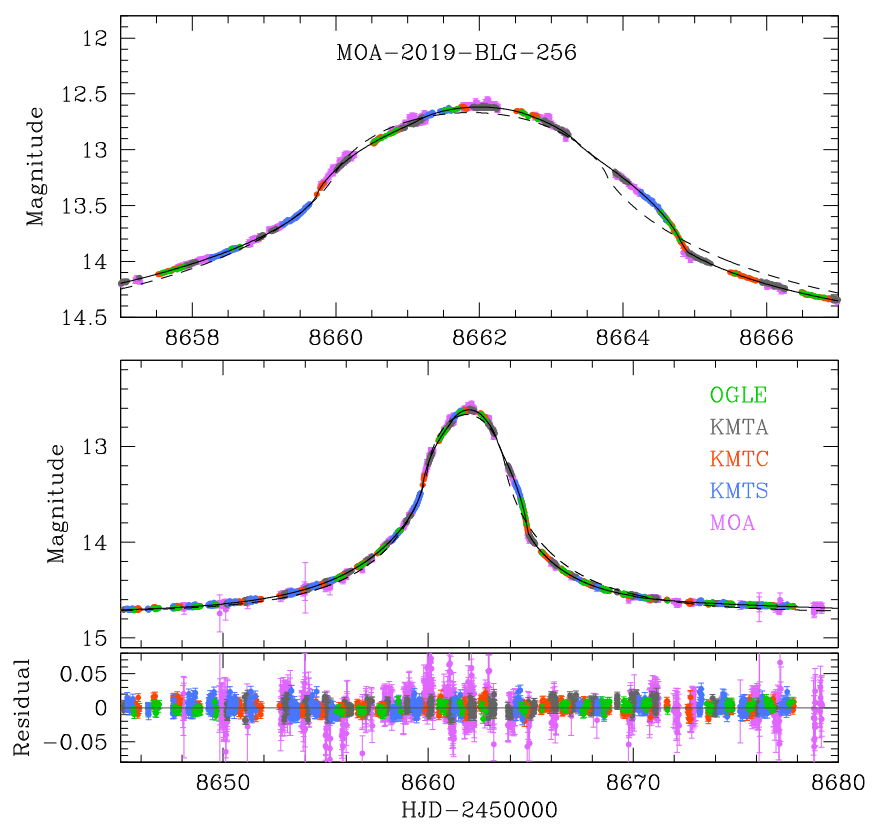

Figure 3. Lightcurve of MOA-2019-BLG-256. The solid and dashed curves represent the model curves based on 2L1S and 1L1S modeling, respectively. For both models, finite-source effects are considered.

giant stars of a similar spectral type ranging from $\mathrm{K} 0$ to $\mathrm{K} 3$. Based on the stellar type, we set the limb-darkening coefficients as $\Gamma_{I}=0.41$, and $\Gamma_{\mathrm{MOA}} \sim\left(\Gamma_{I}+\Gamma_{R}\right) / 2=0.52$ by adopting the values from Claret (2000) under the assumption that $v_{\text {turb }}=$ $2 \mathrm{~km} \mathrm{~s}^{-1}, \log \left(g / g_{\odot}\right)=-2.4$, and $T_{\text {eff }}=4500 \mathrm{~K}$.

We search for the best-fit lensing parameters using the combination of downhill and grid search approaches. For events produced by single lenses, i.e., MOA-2017-BLG-147, and MOA-2017-BLG-241, lensing parameters are searched for by minimizing $\chi^{2}$ using the algorithm of the Markov Chain Monte Carlo (MCMC) method with an adaptive step-size Gaussian sample (Doran \& Mueller 2004). In this search, the initial values of the parameters are given considering the time of the peak, $t_{0}$, peak magnification, $A_{\text {peak }}$, duration of the event, and duration of finite-source anomaly, $\Delta t$. For 1L1S events affected by severe finite-source effects, the peak magnification is approximated as $A_{\text {peak }} \sim\left(1+4 / \rho^{2}\right)^{1 / 2}$ (Maeder 1973;
Agol 2003; Riffeser et al. 2006; Han 2016). For the 2L1S event, i.e., MOA-2019-BLG-256, the analysis is done in two steps. In the first step, we conduct a grid search for the binary lensing parameters $s$ and $q$, while the other parameters are searched for using the MCMC downhill approach. In the second step, we refine the solution(s) found from the initial grid search by allowing all parameters including $s$ and $q$ to vary. Modeling 2L1S events often results in multiple solutions caused by various types of degeneracy. For MOA-2019-BLG256 , we find a unique solution without any degeneracy. We also check the possible degeneracy between binary lens (2L1S) and binary-source (1L2S) solutions. We find that the 1L2S interpretation does not explain the observed anomaly.

In Table 2, we list the best-fit lensing parameters of the individual events. For the 2L1S event MOA-2019-BLG-256, we present three event timescales of $\left(t_{\mathrm{E}}, t_{\mathrm{E}, 1}, t_{\mathrm{E}, 2}\right)$, in which $t_{\mathrm{E}}$ is the timescale corresponding to the total mass of the binary lens, while $t_{\mathrm{E}, 1}$ and $t_{\mathrm{E}, 2}$ represent the timescales corresponding to the masses of individual lens components, i.e., $t_{\mathrm{E}, 1}=\sqrt{1 /(1+q)} t_{\mathrm{E}}$ and $t_{\mathrm{E}, 2}=\sqrt{q /(1+q)} t_{\mathrm{E}}$. The uncertainties of the parameters are estimated as the standard deviation of the points in the MCMC chain, in which the number of points in each MCMC chain is $3 \times 10^{4}$. It is found that the estimated event timescales are very short, ranging from $t_{\mathrm{E}} \sim 1.9$ days to $\sim 6.4$ days according to the timescales corresponding to the individual lens components. It is also found that the normalized source radii are very big, ranging from $\rho \sim 0.14$ to $\sim 0.29$. Also listed in the table are the flux values of the source, $f_{\mathrm{s} \text {, OGLE }}$, and blend, $f_{\mathrm{b}, \text { OGLE }}$, estimated according to the OGLE scale, in which $f=1$ for an $I=18.0$ mag star. The dominance of the source flux over the blend flux indicates that blending is negligible for all events.

In Figure 4, we present lens system configuration of the 2L1S event MOA-2019-BLG-256. The blue dot marked by $M_{1}$ and $M_{2}$ denote the positions of the binary lens components. The mass ratio between the lens components is $q=M_{2} / M_{1}=0.835 \pm 0.003$, and they are separated in projection by $s=1.968 \pm 0.002$. The cuspy curves represent the caustic. Because the separation between $M_{1}$ and $M_{2}$ is greater than $\theta_{\mathrm{E}}$, i.e., $s>1.0$, the caustic is composed of two segments, which are located close to the individual lens components. The line with an arrow represents the source trajectory. The orange circle on the source trajectory represents the source position at the time of the peak magnification and the size of the circle is scaled to the caustic size. It is found that the size 


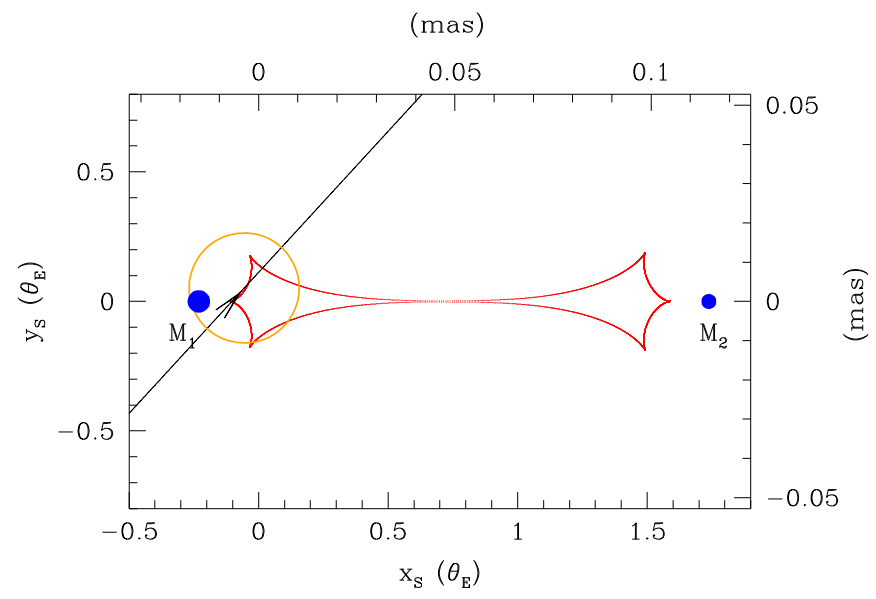

Figure 4. Lens system configuration of the 2L1S event MOA-2019-BLG-256. The two blue dots, marked by $M_{1}$ and $M_{2}$, represent the positions of the lens components and the cuspy closed figure is the caustic. The line with an arrow is the source trajectory. The orange circle on the source trajectory represents the source position at the time of the peak magnification and the size of the circle is scaled to the caustic size. We note that the left and lower sides represent lengths scaled to $\theta_{\mathrm{E}}$ and the right and upper sides represent lengths in milliarcsec (mas).

of the source is similar to that of the caustic located close to $M_{1}$. The source approaches and crosses the caustic multiple times. For general events with a source much smaller than a caustic, sharp spike features appear in the lensing lightcurve at the times of the individual caustic approaches and crossings. For MOA-2019BLG-256, such a spike feature does not appear in the lightcurve due to the severe attenuation of the lensing magnification by finitesource effects.

\section{Angular Einstein Radius}

For the additional constraint of the lens mass, we estimate the angular Einstein radii of the events. The angular Einstein radius is estimated from the combination of the normalized source radius $\rho$ and the angular source radius $\theta_{*}$ by $\theta_{\mathrm{E}}=\theta_{*} / \rho$. The value of $\rho$ is measured from modeling the parts of the lightcurve affected by finite-source effects. The angular source radius is estimated from the dereddened color $(V-I)_{0}$ and brightness $I_{0}$ of the source star.

We use the method of Yoo et al. (2004) to estimate $(V-I)_{0}$ and $I_{0}$. Following this method, we first measure the instrumental color $V-I$ and magnitude $I$ of the source and place the source location on the instrumental color-magnitude diagram (CMD) that is constructed in the same photometric system as that used to process data for the $V-I$ and $I$ measurements. We then measure the offsets in color, $\Delta(V-I)$, and magnitude, $\Delta I$, from the centroid of red giant clump (RGC) with a location on the instrumental CMD of $(V-I, I)_{\mathrm{RGC}}$. Since the source star is located in the bulge with a similar distance to those of red giants, the source and red giant stars experience similar reddening and extinction. Under the assumption of the same reddening and extinction, then, the dereddened source color and magnitude are obtained from the offsets in color and magnitude by

$$
(V-I, I)_{0}=(V-I, I)_{\mathrm{RGC}, 0}+\Delta(V-I, I),
$$

where $(V-I, I)_{\mathrm{RGC}, 0}$ represent the known dereddened color and magnitude of the RGC centroid. For the rereddened color of the RGC centroid, we adopt $(V-I)_{\mathrm{RGC}, 0}=1.06$ from Bensby et al.

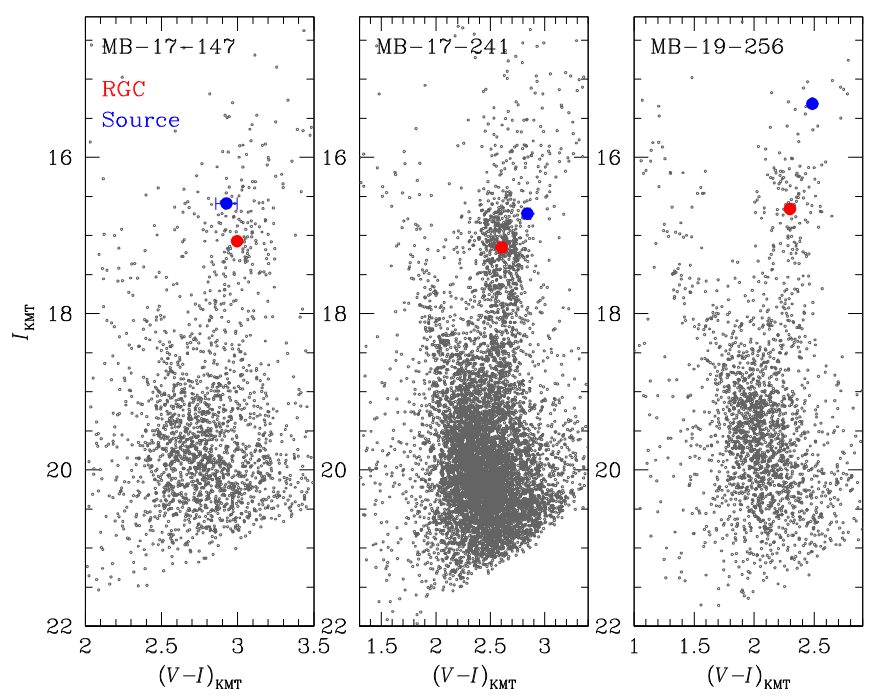

Figure 5. Source locations (blue dots) with respect to the centroids of red giant clump (RGC, red dots) in the instrumental color-magnitude diagrams constructed based on the pyDIA photometry of the KMTC data set.

Table 3

Best-fit Lensing Parameters

\begin{tabular}{lccc}
\hline \hline \multicolumn{1}{c}{ Parameter } & $\begin{array}{c}\text { MOA-2017- } \\
\text { BLG-147 }\end{array}$ & $\begin{array}{c}\text { MOA-2017- } \\
\text { BLG-241 }\end{array}$ & $\begin{array}{c}\text { MOA-2019- } \\
\text { BLG-256 }\end{array}$ \\
\hline$V-I$ & $2.93 \pm 0.07$ & $2.84 \pm 0.03$ & $2.48 \pm 0.01$ \\
$I$ & $16.59 \pm 0.01$ & $16.72 \pm 0.01$ & $15.32 \pm 0.01$ \\
$(V-I, I)_{\mathrm{RGC}}$ & $(3.00,17.03)$ & $(2.61,17.15)$ & $(2.30,16.67)$ \\
$(V-I, I)_{\mathrm{RGC}, 0}$ & $(1.06,14.51)$ & $(1.06,14.65)$ & $(1.06,14.30)$ \\
$(V-I)_{0}$ & $0.99 \pm 0.07$ & $1.30 \pm 0.03$ & $1.25 \pm 0.01$ \\
$I_{0}$ & $14.03 \pm 0.01$ & $14.22 \pm 0.01$ & $12.95 \pm 0.10$ \\
$\theta_{*}(\mu$ as $)$ & $6.94 \pm 0.69$ & $8.06 \pm 0.60$ & $14.07 \pm 0.99$ \\
$\theta_{\mathrm{E}}(\mathrm{mas})$ & $0.051 \pm 0.005$ & $0.028 \pm 0.004$ & $0.066 \pm 0.005$ \\
$\theta_{\mathrm{E}, 1(\mathrm{mas})}$ & $\ldots$ & $\ldots$ & $0.049 \pm 0.004$ \\
$\theta_{\mathrm{E}, 2}(\mathrm{mas})$ & $\ldots$ & $\ldots$ & $0.045 \pm 0.003$ \\
$\mu$ (mas yr $\left.^{-1}\right)$ & $6.89 \pm 0.69$ & $5.42 \pm 0.83$ & $2.76 \pm 0.19$ \\
Spectral type & K0III & K3III & K3III \\
\hline
\end{tabular}

Note. For the 2L1S event MOA-2019-BLG-256, $\theta_{\mathrm{E}}$ is the angular Einstein radius corresponding to the total mass of the binary lens, and $\theta_{\mathrm{E}, 1}$ and $\theta_{\mathrm{E}, 2}$ represent the Einstein radii corresponding to the masses of individual lens components.

(2013). For the dereddened brightness at the Galactic center, we adopt $I_{\mathrm{RGC}, 0}=14.51$ (Nataf et al. 2013). Since the bulge is bar-shaped, the brightness $I_{\mathrm{RGC}, 0}$ slightly varies depending on the source location due to the tilt of the triaxial bulge with respect to the line of sight. For events occurring at different locations, $I_{\mathrm{RGC}, 0}$ is estimated by considering the distance offset from the Galactic center as $\Delta d=\cos l+\sin l(\cos \phi / \sin \phi)$, where $\phi \sim 40^{\circ}$ represents the bar orientation angle (Nataf et al. 2013). In Table 3 , we list $(V-I, I)_{\mathrm{RGC}, 0}$ toward the fields of the individual events.

In Figure 5, we mark the positions of the source stars of the individual events with respect to the RGC centroids on the instrumental CMDs. The CMDs are obtained using the pyDIA photometry of the KMTC data set, and the source color and brightness are measured based on the lightcurve data processed 
using the same photometry code. Since the dereddened source color and brightness are determined from the offsets $\Delta(V-I)$ and $\Delta I$, i.e., Equation (4), we note that the absolute values of the instrumental color and magnitude are irrelevant with the $(V-I)_{0}$ and $I_{0}$ determinations as long as the offsets are measured in the CMD constructed using the same photometry system as that used to produce the lightcurve data from which $(V-I, I)$ are measured.

In Table 3, we list the colors and magnitudes of the source, $(V-I, I)$, and the RGC centroid, $(V-I, I)_{\mathrm{RGC}}$, on the instrumental CMD. With $(V-I, I)_{\mathrm{RGC}, 0}$ together with the measured offsets $\Delta(V-I, I)$, the dereddened colors and magnitudes of the source stars are computed using Equation (4) and listed in Table 3 . The ranges of the $I$-band magnitudes, $13.0 \lesssim I_{0} \lesssim 14.2$, and the color, $1.0 \lesssim(V-\mathrm{I})_{0} \lesssim 1.3$, indicate that the source stars of the events are bulge giant stars of a similar spectral type, ranging from $\mathrm{K} 0$ to $\mathrm{K} 3$.

With the estimated dereddened color and magnitude, we then determine the angular source radii. This is done first by converting the measured $V-I$ color into $V-K$ color using the color-color relation of Bessell \& Brett (1988) and then estimating $\theta_{*}$ using the $(V-K) / \theta_{*}$ relation of Kervella et al. (2004). Once the source radius is estimated, the angular Einstein radius is determined by $\theta_{\mathrm{E}}=\theta_{*} / \rho$.

In Table 3 , we list the estimated values of $\theta_{*}$ and $\theta_{\mathrm{E}}$ for the individual events. For the 2L1S event MOA-2019-BLG-256, we additionally present the Einstein radii corresponding to the masses of the individual lens components, $\theta_{\mathrm{E}, 1}$ and $\theta_{\mathrm{E}, 2}$, similar to the presentation of $t_{\mathrm{E}, 1}$ and $t_{\mathrm{E}, 2}$ in Table 2. Also listed are the relative lens-source proper motions estimated by

$$
\mu=\frac{\theta_{\mathrm{E}}}{t_{\mathrm{E}}}
$$

It is found that the angular Einstein radii are in the range of 0.028 mas $\lesssim \theta_{\mathrm{E}} \lesssim 0.051$ mas. These values are more than an order smaller than $\sim 0.5$ mas of typical lensing events produced by low-mass lenses located roughly halfway between the observer and source. The estimated relative lens-source proper

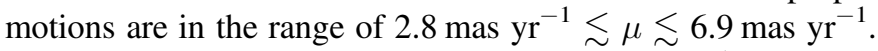
These values are smaller or similar to $\sim 5 \mathrm{mas} \mathrm{yr}^{-1}$ of typical lensing events. This indicates that the very short timescales of the analyzed events are not caused by unusually high relative lens-source proper motions, but more likely to be caused by the low masses of the lenses.

\section{Nature of Lenses}

For the characterization of the lenses, we estimate the physical lens parameters of the lens mass $M$ and distance $D_{\mathrm{L}}$. In order to uniquely determine $M$ and $D_{\mathrm{L}}$, it is required to determine both the angular Einstein radius $\theta_{\mathrm{E}}$ and the microlens parallax $\pi_{\mathrm{E}}$, which are related to the lens mass and distance by

$$
M=\frac{\theta_{\mathrm{E}}}{\kappa \pi_{\mathrm{E}}} ; \quad D_{\mathrm{L}}=\frac{\mathrm{au}}{\pi_{\mathrm{E}} \theta_{\mathrm{E}}+\pi_{\mathrm{S}}},
$$

where $\pi_{\mathrm{S}}=\mathrm{au} / D_{\mathrm{S}}$ is the parallax of the source. For all the analyzed events, the angular Einstein radii are securely measured from the detections of finite-source effects. The microlens parallax is measurable by detecting deformations in lensing lightcurves caused by the deviation of the source motion from rectilinear due to the change of the observer's
Table 4

Source Proper Motion

\begin{tabular}{ccc}
\hline \hline Event & $\mu_{\text {R.A. }}\left(\mathrm{mas} \mathrm{yr}^{-1}\right)$ & $\mu_{\text {decl. }}\left(\mathrm{mas} \mathrm{yr}^{-1}\right)$ \\
\hline MOA-2017-BLG-147 & $-5.348 \pm 0.335$ & $-7.694 \pm 0.272$ \\
MOA-2017-BLG-241 & $-3.775 \pm 0.450$ & $-4.049 \pm 0.396$ \\
MOA-2019-BLG-256 & $-2.299 \pm 0.170$ & $-6.973 \pm 0.134$ \\
\hline
\end{tabular}

Note. $\mu_{\text {R.A. }}$ and $\mu_{\text {decl. }}$ denote the proper motions in R.A. and decl. directions, respectively.

position induced by the orbital motion of Earth around the Sun (Gould 1992), e.g., OGLE-2016-BLG-0156 (Jung et al. 2019). The microlens parallax cannot be measured through this annual microlens parallax channel for any of the events because the timescales of the events are too short to yield measurable deviations in the lensing lightcurves. Besides this channel, the microlens parallax can be measured from simultaneous observations of lensing events using ground-based telescopes and a space-based satellite: "space-based microlens parallax" (Refsdal 1966; Gould 1994b), e.g., OGLE-2015-BLG-0966 (Street et al. 2016). See a more detailed discussion about the space-based microlens parallax measurements in Section 7. Unfortunately, space-based observation has not been conducted for any of the events. We, therefore, estimate the physical lens parameters by conducting Monte Carlo simulations of events with the constraints of the measured event timescales together with the angular Einstein radii.

Simulations of lensing events are conducted based on the prior models of the physical and dynamical distributions of astronomical objects in the Galaxy and their mass function. For the threedimensional physical matter distributions, we use the Han \& Gould (2003) model, in which the disk matter density follows a double-exponential distribution and the bulge matter density is described by a triaxial bulge. For the details of the distributions, see Sections 2.1 and 2.2 of Han \& Gould (2003). For the model of the relative lens-source motion, we adopt the nonrotating barred bulge model described in Table 1 of Han \& Gould (1995). For the mass function of stars and BDs, we adopt separate distributions for disk and bulge lenses, for which the initial mass function and the present day mass function of Chabrier (2003) are adopted, respectively. Based on these distributions, we include stellar remnants, i.e., black holes, neutron stars, and white dwarfs, in the mass function by adopting the Gould (2000) model. With these models, we conduct Monte Carlo simulations to produce numerous $\left(4 \times 10^{7}\right)$ artificial lensing events. We then construct the probability distribution of the physical parameters for events with timescales and Einstein radii within the ranges of the measured values and estimate the physical parameters and their uncertainties. We obtain two sets of probability distributions, in which one set of distributions are obtained with only the constraint of $t_{\mathrm{E}}$, whereas the other set of distributions is obtained with the combined $t_{\mathrm{E}}$ and $\theta_{\mathrm{E}}$ constraints. We note that the source stars of all the events are bright and their proper motions are measured by Gaia (Gaia Collaboration et al. 2018). In Table 4, we list the proper motions of the individual events. We consider the measured proper motions of the source stars in the analysis.

In Figure 6, we present the probability distributions of the physical lensing parameters obtained from the Monte Carlo analysis. For each event, the left and middle panels show the probability distributions of the lens mass and the lens-source 

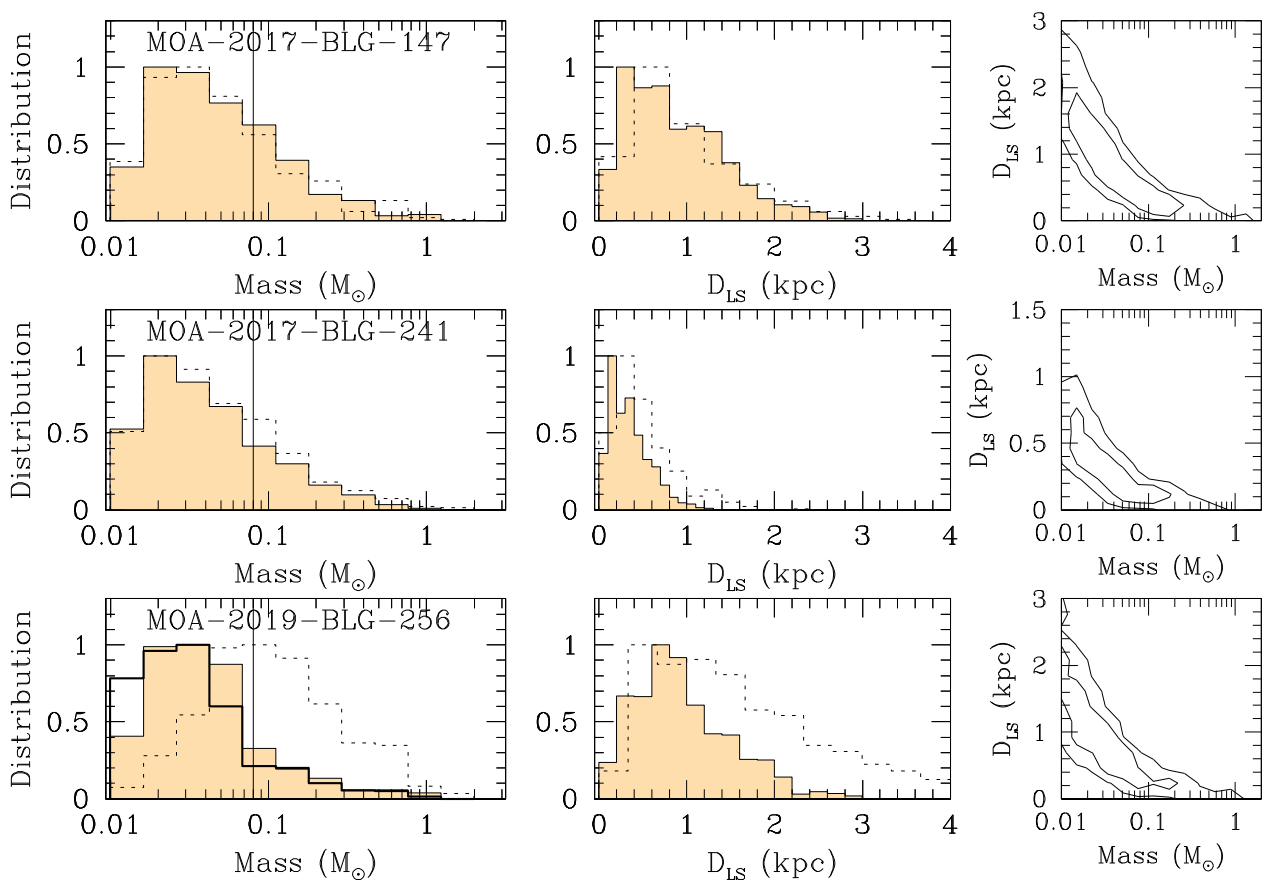

Figure 6. Probability distributions of the lens mass $(M)$ and the lens-source separation $\left(D_{\mathrm{LS}}\right)$ obtained from the Monte Carlo simulations of Galactic events. In each panel, the solid curve is the probability distribution obtained with the combined $t_{\mathrm{E}}+\theta_{\mathrm{E}}$ constraint, whereas the dotted curve is obtained using the constraint of only $t_{\mathrm{E}}$. The vertical line in each left panel indicates the boundary between stars and BDs, i.e., $0.08 M_{\odot}$. For the 2L1S event MOA-2019-BLG-256, the mass distribution for the heavier lens component, $M_{1}$, is presented as a shade histogram, while the distribution for the lower mass lens component, $M_{2}$, is presented as a solid curve. The right panels show the probability distribution in the $M-D_{\mathrm{LS}}$ plane and the contours represent the $1 \sigma$ and $2 \sigma$ ranges.

Table 5

Physical Lens Parameters

\begin{tabular}{cccc}
\hline \hline Event & $M_{1}\left(M_{\odot}\right)$ & $M_{2}\left(M_{\odot}\right)$ & $D_{\mathrm{LS}}(\mathrm{kpc})$ \\
\hline MOA-2017-BLG-147 & $0.051_{-0.027}^{+0.100}$ & $\ldots$ & $0.87_{-0.45}^{+0.67}$ \\
MOA-2017-BLG-241 & $0.044_{-0.023}^{+0.090}$ & $\ldots$ & $0.36_{-0.18}^{+0.28}$ \\
MOA-2019-BLG-256 & $0.046_{-0.023}^{+0.067}$ & $0.038_{-0.019}^{+0.056}$ & $0.94_{-0.46}^{+0.62}$ \\
\hline
\end{tabular}

Note. For the 2L1S event MOA-2019-BLG-256, $M_{1}$ and $M_{2}$ denote the masses of the individual lens components.

separation $\left(D_{\mathrm{LS}}\right)$, respectively. The right panels show the probability distribution in the $M-D_{\mathrm{LS}}$ plane and the contours represent the $1 \sigma$ and $2 \sigma$ ranges. We note that the lenses are located very close to the source in all cases of the events and thus we present the distribution of $D_{\mathrm{LS}}$ instead of $D_{\mathrm{L}}$. The solid and dotted curves represent the distributions obtained with $t_{\mathrm{E}}+\theta_{\mathrm{E}}$ and $t_{\mathrm{E}}$ constraints, respectively. In Table 5, we list the estimated physical lens parameters. For the 2L1S event MOA2019-BLG-256, we list the masses of both lens components, i.e., $M_{1}$ and $M_{2}$. The presented value of each parameter is estimated as the median of the probability distribution and the lower and upper uncertainties are estimated as the $16 \%$ and $84 \%$ of the distribution, respectively.

We find that the lenses of all events share similar properties that they are very likely to be substellar objects located very close to the source stars. From the Bayesian analysis, it is estimated that the masses of the lenses are $0.051_{-0.027}^{+0.100} M_{\odot}$, $0.044_{-0.023}^{+0.090} M_{\odot}$, and $0.046_{-0.023}^{+0.067} M_{\odot} / 0.038_{-0.019}^{+0.056} M_{\odot}$ for MOA2017-BLG-147L, MOA-2017-BLG-241L, and MOA-2019BLG-256LAB, respectively. The probability for the lens mass smaller than the lower limit for the mass of a star is about $80 \%$ for all events. The lenses of the individual events are located at the locations with the distances from the source of $D_{\mathrm{LS}}=$ $0.87_{-0.45}^{+0.67} \mathrm{kpc}, 0.36_{-0.18}^{+0.28} \mathrm{kpc}$, and $0.94_{-0.46}^{+0.62} \mathrm{kpc}$. The estimated lens masses and locations indicate that the lenses of the events are bulge BDs located close to the source stars. We note that MOA-2019-BLG-256LAB is the fifth microlensing BD binary followed by OGLE-2009-BLG-151L, OGLE-2011-BLG0420L (Choi et al. 2013), MOA-2016-BLG-231L (Chung et al. 2019), and OGLE-2016-BLG-1469L (Han et al. 2017).

It is found that the additional constraint provided by the angular Einstein radius helps to reveal the substellar nature of the lenses. For MOA-2017-BLG-147 and MOA-2017-BLG241 , the probability distributions of $M$ and $D_{\mathrm{LS}}$ with the additional constraint of $\theta_{\mathrm{E}}$ are not much different from the distributions obtained with only the $t_{\mathrm{E}}$ constraint, indicating that the additional constraint of $\theta_{\mathrm{E}}$ is not very strong. However, for MOA-2019-BLG-256, the additional constraint of $\theta_{\mathrm{E}}$ substantially shifts the most probable lens mass and location toward lower masses and closer to the source, respectively. For the former two events, the event timescales, $t_{\mathrm{E}}<2.7$ days, are very short and thus the timescale alone constrains that the lens is likely to be a substellar object. On the other hand, the event timescales of MOA-2019-BLG-256, $t_{\mathrm{E}} \sim 8.7$ days, is relatively long and the $\mathrm{BD}$ nature of the lens can be constrained with the additional constraint of the very small $\theta_{\mathrm{E}}$. The very small $\theta_{\mathrm{E}}$ values also tightly constrain the lens locations, i.e., very close to the source, because $\theta_{\mathrm{E}} \propto\left(D_{\mathrm{LS}} / D_{\mathrm{L}} D_{\mathrm{S}}\right)^{1 / 2}$.

\section{Discussion}

Although the probability of the lenses to be BDs is high, the ranges of the lens masses estimated from the Bayesian analysis are rather big. To firmly identify the BD nature of the lenses, it is desirable to uniquely determine the lens masses by additionally measuring the values of the microlens parallax. 
Table 6

Projected Einstein Radius

\begin{tabular}{ccccc}
\hline \hline Event & $r_{\mathrm{E}}(\mathrm{au})$ & $\tilde{r}_{\mathrm{E}}(\mathrm{au})$ & $D_{\perp}(\mathrm{au})$ & $\tilde{r}_{\mathrm{E}} / D_{\perp}(\mathrm{au})$ \\
\hline MOA-2017-BLG-147 & 0.36 & 3.3 & 1.59 & 0.48 \\
MOA-2017-BLG-241 & 0.21 & 4.8 & 1.59 & 0.33 \\
MOA-2019-BLG-256 & 0.35 & 2.9 & 1.73 & 0.60 \\
\hline
\end{tabular}

Note. $\tilde{r}_{\mathrm{E}}$ denotes the physical Einstein radius projected onto the plane of the observer and $D_{\perp}$ represents the projected Earth-Spitzer separation as seen from the lens-source line of sight.

We point out that the microlens parallax values and thus the lens masses of the events could have been uniquely determined if the events had been observed using a satellite separated from Earth by a substantial fraction of an au. Space-based microlens parallax measurement is optimized when the projected Earthsatellite separation as seen from the lens-source line of sight (projected satellite separation), $D_{\perp}$, comprises an important portion of the physical Einstein radius projected onto the plane of the observer (projected Einstein radius), $\tilde{r}_{\mathrm{E}}=\left(D_{\mathrm{S}} / D_{\mathrm{LS}}\right) r_{\mathrm{E}}$. Here $r_{\mathrm{E}}=D_{\mathrm{L}} \theta_{\mathrm{E}}$ represents the physical Einstein radius. If $D_{\perp} \gg \tilde{r}_{\mathrm{E}}$, the lensing magnifications observed by ground-based telescopes would be difficult to observe with a space-based satellite because the impact parameter of the lens-source approach seen from the satellite would be too big to induce lensing magnifications. If $D_{\perp} \ll \tilde{r}_{\mathrm{E}}$, in contrast, the difference between the two lensing lightcurves obtained from the ground- and space-based observations would be too small to securely measure $\pi_{\mathrm{E}}$.

Considering the Spitzer telescope as an example of a satellite in a heliocentric orbit, we estimate the values of $r_{\mathrm{E}}, \tilde{r}_{\mathrm{E}}$, and $D_{\perp}$ and list them in Table 6. We note that the projected Einstein radius $\tilde{r}_{\mathrm{E}}$ is much bigger than $r_{\mathrm{E}}$ because $\tilde{r}_{\mathrm{E}}$ is inversely proportional to the lens-source distance, i.e., $\tilde{r}_{\mathrm{E}}=\left(D_{\mathrm{S}} / D_{\mathrm{LS}}\right) r_{\mathrm{E}}$, and the lens-source separations are very small for the analyzed events. We also list the ratios of $D_{\perp} / \tilde{r}_{\mathrm{E}}$ corresponding to the Spitzer telescope locations at the times of the events. The ratios are in the range of $0.3 \lesssim D_{\perp} / \tilde{r}_{\mathrm{E}} \lesssim 0.6$, which are optimal ratios for secure $\pi_{\mathrm{E}}$ measurements.

Spitzer observation could not be conducted for any of the events because the current Spitzer microlensing campaign (Calchi Novati et al. 2015) has been conducted in a follow-up mode together with the fact that the timescales of the events are very short. According to the protocol of the Spitzer sample selection (Yee et al. 2015), very short-timescale events are unlikely to be selected because immediate follow-up observation is difficult due to the relatively long period (a week) of uploading observation sequences and the time required to prepare the sequences. These difficulties of observing shorttimescale events can be overcome if space-based observations are carried in a survey mode simultaneously with a groundbased survey. Another important reason for the difficulty of observing the events is the short time window, 40 days, through which the bulge field is observable simultaneously from Spitzer and from the ground. The Spitzer window ran during 7927-7969 and 8671-8712 in the 2017 and 2019 seasons, respectively. As a result, all of the events were at (or nearly at) baseline by the time Spitzer observations started.

\section{Summary and Conclusion}

We investigated strong candidate BD-lens events found from the search for lensing events not only with short timescales but also with very small angular Einstein radii. By imposing the criteria of $t_{\mathrm{E}} \lesssim 6$ days and $\theta_{\mathrm{E}} \lesssim 0.05$ mas for events detected since the 2016 season, we found three events including MOA2017-BLG-147, MOA-2017-BLG-241, and MOA-2019-BLG256 , in which the lens of the last event is a binary. By measuring the event timescales and angular Einstein radii from lightcurve modeling followed by Bayesian analyses of the events with the combined constraint of $t_{\mathrm{E}}$ and $\theta_{\mathrm{E}}$, we estimated that the lens masses of the individual events were $0.051_{-0.027}^{+0.100} M_{\odot}$, $0.044_{-0.023}^{+0.090} M_{\odot}$, and $0.046_{-0.023}^{+0.067} M_{\odot} / 0.038_{-0.019}^{+0.056} M_{\odot}$. We pointed out that uniquely determining lens masses of short-timescale events by additionally measuring microlens parallax values required survey-mode space-based observation using a satellite in a heliocentric orbit.

Work by C.H. was supported by the grants of the National Research Foundation of Korea (2017R1A4A1015178 and 2019R1A2C2085965). Work by A.G. was supported by US NSF grant AST-1516842 and by JPL grant 1500811. A.G. received support from the European Research Council under the European Union's Seventh Framework Programme (FP 7) ERC grant Agreement No. [32103]. The OGLE project has received funding from the National Science Centre, Poland, grant MAESTRO 2014/14/A/ST9/00121 to AU. This research has made use of the KMTNet system operated by the Korea Astronomy and Space Science Institute (KASI) and the data were obtained at three host sites of CTIO in Chile, SAAO in South Africa, and SSO in Australia. The MOA project is supported by JSPS KAKENHI grant Nos. JSPS24253004, JSPS26247023, JSPS23340064, JSPS15H00781, JP17H02871, and JP16H06287. Y.M. acknowledges the support by the grant JP14002006. D.P.B., A.B., and C.R. were supported by NASA through grant NASA-80NSSC18K0274. The work by C.R. was supported by an appointment to the NASA Postdoctoral Program at the Goddard Space Flight Center, administered by USRA through a contract with NASA. N.J.R. is a Royal Society of New Zealand Rutherford Discovery Fellow.

\section{ORCID iDs}

Cheongho Han (D) https://orcid.org/0000-0002-2641-9964 Valerio Bozza (iD https://orcid.org/0000-0003-4590-0136 Sun-Ju Chung (iD https://orcid.org/0000-0001-6285-4528 Kyu-Ha Hwang (i) https://orcid.org/0000-0002-9241-4117 Yoon-Hyun Ryu (iD https://orcid.org/0000-0001-9823-2907 In-Gu Shin (i) https://orcid.org/0000-0002-4355-9838 Yossi Shvartzvald (iD https://orcid.org/0000-0003-1525-5041 Jennifer C. Yee (1D https://orcid.org/0000-0001-9481-7123 Weicheng Zang (iD https://orcid.org/0000-0001-6000-3463 Richard W. Pogge (D) https://orcid.org/0000-0003-1435-3053 Jan Skowron (1) https://orcid.org/0000-0002-2335-1730 Paweł Pietrukowicz (10) https://orcid.org/0000-0002-2339-5899 Krzysztof Ulaczyk (i) https://orcid.org/0000-0001-6364-408X Patryk Iwanek (i) https://orcid.org/0000-0002-6212-7221 David P. Bennett (iD https://orcid.org/0000-0001-8043-8413 Akihiko Fukui (iD https://orcid.org/0000-0002-4909-5763 Yoshitaka Itow (iD https://orcid.org/0000-0002-8198-1968 Iona Kondo (iD https://orcid.org/0000-0002-3401-1029 Naoki Koshimoto (iD https://orcid.org/0000-0003-2302-9562 Shota Miyazaki (iD https://orcid.org/0000-0001-9818-1513 Clément Ranc (iD https://orcid.org/0000-0003-2388-4534 Nicholas J. Rattenbury (iD https://orcid.org/0000-00015069-319X

Daisuke Suzuki (iD https://orcid.org/0000-0002-5843-9433 


\section{References}

Agol, E. 2003, ApJ, 594, 449

Alard, C., \& Lupton, R. H. 1998, ApJ, 503, 325

Albrow, M. 2017, MichaelDAlbrow/pyDIA: Initial Release on Github, Version, v1.0.0, Zenodo, doi:10.5281/zenodo.268049

Albrow, M., Horne, K., Bramich, D. M., et al. 2009, MNRAS, 397, 2099

Bensby, T., Yee, J. C., Feltzing, S., et al. 2013, A\&A, 549, 147

Bessell, M. S., \& Brett, J. M. 1988, PASP, 100, 1134

Bond, I. A., Abe, F., Dodd, R. J., et al. 2001, MNRAS, 327, 868

Calchi Novati, S., Gould, A., Udalski, A., et al. 2015, ApJ, 804, 20

Chabrier, G. 2003, ApJL, 586, L133

Chabrier, G., Johansen, A., Janson, M., \& Rafikov, R. 2014, in Protostars and Planets VI, Vol. 914 ed. H. Beuther et al. (Tucson: Univ. Arizona Press), 619

Choi, J.-Y., Han, C., Udalski, A., et al. 2013, ApJ, 768, 129

Choi, J.-Y., Shin, I.-G., Park, S.-Y., et al. 2012, ApJ, 751, 41

Chung, S.-J., Gould, A., Skowron, J., et al. 2019, ApJ, 871, 179

Claret, A. 2000, A\&A, 363, 1081

Dong, S., DePoy, D. L., Gaudi, B. S., et al. 2006, ApJ, 642, 842

Doran, M., \& Mueller, C. M. 2004, JCAP, 9, 3

Gaia Collaboration, Brown, A. G. A., Vallenari, A., et al. 2018, A\&A, 616, A1

Gould, A. 1992, ApJ, 392, 442

Gould, A. 1994a, ApJL, 421, L71

Gould, A. 1994b, ApJL, 421, L75

Gould, A. 2000, ApJ, 535, 928
Han, C. 2016, ApJ, 820, 53

Han, C., \& Gould, A. 1995, ApJ, 447, 53

Han, C., \& Gould, A. 2003, ApJ, 592, 172

Han, C., Udalski, A., Sumi, T., et al. 2017, ApJ, 843, 59

Jung, Y. Kil., Han, C., Bond, I. A., et al. 2019, ApJ, 872, 175J

Kervella, P., Thévenin, F., Di Folco, E., \& Ségransan, D. 2004, A\&A, 426, 29

Kim, S.-L., Lee, C.-U., Park, B.-G., et al. 2016, JKAS, 49, 37

Maeder, A. 1973, A\&A, 26, 215

McLean, I. S., McGovern, M. R., Burgasser, A. J., et al. 2003, ApJ, 596, 561

McLean, I. S., Prato, L., McGovern, M. R., et al. 2007, ApJ, 658, 1217

Mróz, P., Ryu, Y.-H., Skowron, J., et al. 2018, AJ, 155, 121

Mróz, P., Udalski, A., Bennett, D. P., et al. 2019, A\&A, 622, A201

Nataf, D. M., Gould, A., Fouqué, P., et al. 2013, ApJ, 769, 88

Paczyński, B. 1986, ApJ, 304, 1

Refsdal, S. 1966, MNRAS, 134, 315

Riffeser, A., Fliri, J., Seitz, S., \& Bender, R. 2006, ApJS, 163, 225

Street, R. A., Udalski, A., Calchi Novati, S., et al. 2016, ApJ, 819, 93

Udalski, A., Szymański, M. K., \& Szymański, G. 2015, Acta Astron, 65, 1

Whitworth, A., Bate, M. R., Nordlund, A., Reipurth, B., \& Zinnecker, H. 2007, in Protostars and Planets, Vol. 951 ed. V. B. Reipurth, D. Jewitt, \& K. Keil (Tucson: Univ. Arizona Press), 459

Witt, H. J., \& Mao, S. 1994, ApJ, 430, 505

Woźniak, P. R. 2000, AcA, 50, 421

Yee, J. C., Gold, A., Beichman, C., et al. 2015, ApJ, 810, 155

Yee, J. C., Shvartzvald, Y., Gal-Yam, A., et al. 2012, ApJ, 755, 102

Yoo, J., DePoy, D. L., Gal-Yam, A., et al. 2004, ApJ, 603, 139 